\title{
The Ontological Law of Contradiction and Its Logical Structure
}

\author{
Toshiharu WARAgaI \\ Tokushima University
}

\section{\$1 Introduction}

What is aimed at in this paper is the construction of the basic part of a formal language by means of which we are able to analyse and describe the logicosemantical structure of traditional Ontology. In carrying out the attempt, I required of the language that it have the ability to express the logico-semantical structure of the so-called "ontological law of identity" and the "ontological law of contradiction" as its necessary condition. It is precisely because these two ontological laws are regarded as the most basic statements that express the most fundamental character of primary substances; namely, they are said to express the self-dependency of primary substances which are independent of anything other than themsevles, being dependent solely on themsevles. Primary substances are the very cause thanks to whose existence Ontology is able to subsist as a science.

But we have to admit that the logico-semantical intention and structure are rather ambiguous and unclear, when they are expressed in so-called "everyday langauge". For this reason, I aim in this paper at the construction of a formal language which is able to exhibit explicitely their logico-semantical character.

\section{\$2 The Argument of Descending Chain of Predicates}

According to traditional Ontology, the most basic characteristic of primary substances is that with reference to them

1. the ontological law of identity, and

2. the ontological law of contradiction

hold. These two principles characterise the most basic properties of primary substances. The ontological law of identity is stated in the following form;

O.L.I. $A$ est $A$, while the ontological law of contradiction is stated either in the following form;

O.L.C.1. $A$ non est $B$ et non $B$

or in the following form;

O.L.C.2. $A$ non est non $A$.

What is peculiar to them is that they express a kind of ontological circumstances concerning primary substances by means of a logico-grammatial device. But insofar 
as the logical structure of the logico-grammatical device remains not explicitly explaned, we cannot be immune from the objection that they are nothing more than a play on words. Indeed J. Lukasiewicz once stated;

Zur Klarheit in dieser Frage hat uns erst die symbolische Logik verholfen. Die sogen. "philosophische" Logik ist in dieser Hinsicht nichts mehr als lauter phrasenmacherei. Unter dem principium identitatis wird einmal das Prinzip der Identität, ein anderes Mal der Satz des Widerspruchs verstanden, der Satz des Widerspruchs wird mit dem mangelhaft formlierten Prinzip der doppleten Verneinung " $A$ ist nicht non- $A$ " verwechselt, das Prinzip der Identität, für welches die vieldeutige und zum mindesten ungenaue Formel " $A$ ist $A$ " allgemein verwendet..., wird als "positive Kehrseite" dem Satz des Widerspruchs an die Seite gestellt und mit demselben identifiziert, u.s.f. Für feinere Begriffsunterschiede hat eben die philsophische Logik gar keinen Sinn, weil sie nicht mit scharf begrenzten Begriffen und eindeutig bestimmten Symbolen arbeitet, sondern im Sumpfe der schwankenden und vagen, im alltäglichen Leben verbrauchten Redensarten zerfließt. (1)

My purpose in this paper is to find the most appropriate way of describing these two basic laws. In other words, I aim at some particular logical language in which these two basic laws are describable in the most proper way. So the language I aim at must be able to bring out their logical structure. In this sense, the language I aim at in this paper is rightly philosophical, but at the same time I may well insist that the language is completely immune from Kukasiewicz's objection, because it can be governed by strictly regimented logical rules.

For the purpose of constructing such a logical language, I choose a special device which was used by Aristotle in determining the ontological status of primary substances in terms of logic. His whole argument is to be found in Analytica Posteriora, A, XIX-XXII. There he develops an argument which I want to name the argument of descending chain of predicates, ${ }^{(2)}$ which is both logically and philosophically quite interesting, since the logico-semantical analysis of this argument supplies us with a specific logical apparatus for the analysis of the two ontological laws.

I summarize Aristotle's point quite simply. According to him;

A. $\cdots$ neither the ascending nor the descending series of predication in which a single attribute is predicated of a single subject is infinite ${ }^{(3)}$.

(1) "Uber den Satz von Widerspruch bei Aristoteless", Bulletin international de Académie des Sciences de Cracovie, Classe de Philosophie, 1910, p. 22.

(2) I use the term "predicate" in the sense of "praedioatum", i.e. the term which takes the second position in a sentence of the form "A est $B$ ". So the term " $B$ " is called the predicate of the sentence, while the the term " $\mathrm{A}$ " is called the "subject" of the sentence.

(3) APst., A, XXII, 83b24-26. Ross translation. 
According to Aristotle, we come across a term of specific character, namely a so-called "ultimate subject", when we proceed downwards along a chain of predicates which constitute a proof. Its specific character is that it cannot be predicated to anything except itself, while the other terms can at one time take the position of subject and at another time take the position of predicate. Their logico-grammatical status is relative, while the logico-grammatical status of the ultimate subject is, in a sense, absolute. If we go upwards along the chain, then we come across in fintite steps a term of specific character. Its specific character consists in that it cannot take the position of subject except in one case, i.e. selfpredication. This term is called "maximum genus". All the other terms in this chain of predicates are between these two specific terms. Let me illustlate this logical situation by means of a diagram;

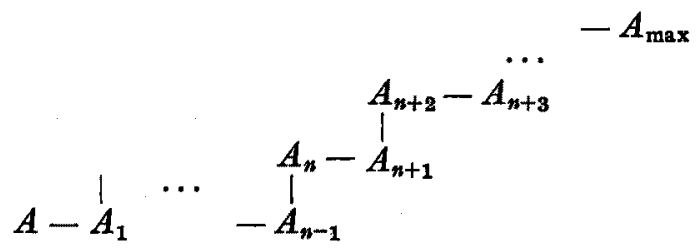

In this diagram, the term " $A_{\max }$ " stands for the maximum genus, and the term " $A$ " stands for the ultimate subject. In the following part of this paper, we shall mainly be concerned with the descending direction of the chain of predicates. Since every predicate can be regarded as a general name, so we may well suppose that every term in the chain belongs to the syntactic category of "general names". To put it in other words, we do not need in this context the syntactic category of "singular names". This supposition can be upheld by the fact taht Aristotle himself seems to treat the names which are normally taken to belong to the syntactic category of singular names as some kind of general names. Aristotle states;

But it has been shown that in these substantial predications neither the ascending predicates nor the descending subject form an infinite series; e.g. neither the series, man is biped, biped is animal, \&c., nor the series predicating animal of man, man of Callias, Callias of a further subject as an element of its essential nature, is infinite. (APst. A, XXII, 83bl-5, Italics by the present author)

(1) Arigtotle maintains; For there is no difference between "the man is recovering" and 'the man recovers', nor between 'the man is walking' or 'cutting' and 'the man walks' or 'cuts'; and similarly in all other cases. (Meta., V, 7, 1017a27-30) Thomas gives a concise commentary on this passage; "Verbum enim quodlibet resolvitur in hoo verbum Est, et participium." (In Meta., L.V, lec. 9, 893) Another passage to be mentioned from Aristotle's is; "for to say 'man walks' is merely equivalent to saying "man is walking'." (De Int., 12, 21b9-10) 
It seems to be obvious that Aristotle treats in this context the name "Callias" as a kind of general name.(1) Taking these circumstances into account, let us suppose that we have only the syntactic category of general names at our disposal. Generally speaking, there is no decisive logical reason for dividing names into two syntactic categories, i.e. that of singular names and that of general names. I take the position that a singular name is a general name whose extension is just one.

Now let us consider the logico-semantic characteristics of the ultimate subject " $A$ ". What is characteristic of " $A$ " is that it cannot take the position of predicate except in the case of self-predication, while it can take the position of subject with respect to all other terms appearing in the chain. If we use so-called "everyday language" for expressing the above mentioned cases of predication, then they will be expressed in the following way;

a) $A$ est $A$,

b) $A$ est $A_{n}$.

As for the other predications, the grammatical form remains the same, i.e.;

c) $A_{i}$ est $A_{j}$

But here we should notice very clearly the distinction between the function of the coupla "est" in a), b)-cases and that in c)-case, though the same expression "est" is used. The "est" in a), b)-cases takes as its grammatical subject only the terms each of which refers to one individual only, while the "est" in c)- case takes as its subject the terms each of which refers to more than one individual indefinitely. Since we are considering in such circumstances where only the syntactic category of general names is at our diposal, supposing that we have no special syntactic category of singualar names, the logico-semantical difference between the predication of a), b)-type and that of c)-type has to be captured as the difference between the logico-semantical function of "est". in a), b)-case and that of the "est" in c)-case. Considering that the "est" in c)-case is reducible to the "est" in a), b)case, and the "est" in a), b)-case constructs the so-called "singular sentence" - its subject directly refers to an individual and something is stated to the individual by means of its predicate - I call the "est" in a), b)-case the purely ontological coupla, and in order to discriminate it also morphologically from the "est" in c)-case, I hereafter write the purely ontological copula as " $\varepsilon$ ". In addition, I call a sentence of the form " $A \varepsilon B$ " a purely ontological sentence.

(1) Cf. Thomas's commentary. He states; "...; neque in deorsum, ut si animal dioatur de homine in eo quod quid est, et homo de Callia, et hoc de quodam alio (supposito quod homo esset genus continens sub se multas species, quarum una esset Callias), non posset sic procedi in infinitum. (In $A P$ st., L.I., lec. XXXIC, 291, Italics by the present author) 


\section{§3 Purely Ontological Copula}

Now the problem passes on to the description of logical features of " $\varepsilon$ ". The characterisation of the purely ontological copula is not very difficult. Indeed it can be done in the following way; since a sentence constructed by means of the purely ontological copula has as its grammatical subject a (general) name that refers to just one individual, so, supposing that we have a sentence " $A \varepsilon B$ ", the term " $A$ " must meet the following conditions;

0.1. some individual is $A$,

0.2. at most one individual is $A$.

If the term " $A$ " meets these two conditions, then the following condition states that the very object referred to by the term " $A$ " is $B$;

0.3. every $\boldsymbol{A}$ is $\boldsymbol{B}$.

Now it soon becomes clear when we look at these three conditions closely that we can formulate the conditions $0.1-0.3$ by means of " $\varepsilon$ " and logical connectives, namely;

$$
\begin{array}{ll}
0.1^{*} & (\exists X)(X \varepsilon A) \\
0.2^{*} & (X, Y)\left(X \varepsilon A_{\wedge} Y \varepsilon A . \supset X \varepsilon Y\right) \\
0.3^{*} & (X)(X \varepsilon A \supset X \varepsilon B)^{(\mathbf{1})}
\end{array}
$$

And, in reverse, if the terms " $A$ " and " $B$ " meet these three conditions $0.1^{*}-0.3$ ", then there holds the following relation between them i.e.;

0.4. the term " $A$ " refers to just one individual and it is $B$, which says;

0.4*. $A \varepsilon B$,

with the result that we reach the following condition which governs the logical feature of the purely ontological copula;

$$
\text { Ax. 0. } A \varepsilon B \equiv .(\exists X)(X \varepsilon A)_{\wedge}(X, Y)\left(X \varepsilon A_{\wedge} Y \varepsilon A . \supset X \varepsilon Y\right)_{\wedge}
$$

$$
(X)(X \varepsilon A \supset X \varepsilon B) \text {. }
$$

It should be noticed that in Ax. O. " $\varepsilon$ " appears on the both sides of the equivalence symbol, and the other symbols appearing in Ax.O. are all logical connectives except the symbols for names. So Ax.0. is not a definition of " $\varepsilon$ ", but an axiom which governs the logical behavior of " $\varepsilon$ ", with the result that we succeeded in drawing the most basic usage of "est" out of its many ambiguous usages. Indeed the "est" used in the sense of c)-case is definable by means of " $\varepsilon$ " in the following way;

(1) For the time being, we may take the referential interpretation of quantifiers. Indeed as long as we quantify only the terms for which the ontological law of identity lholds, we may follow this interpretation, while the logical situation changes if we begin also to quantify the other parts of sentences. Then we shall be in need of another interpretation of quantifiers in order to secure the correspondence between 0.1., 0.2 , 0.3 . and $0.1^{*}, 0.2^{*}, 0.3^{*}$. This point will be disoussed below. 
Def. $\quad A \sqsubset B \equiv(\exists X)(X \varepsilon A)_{\wedge}(X)(X \varepsilon A \supset X \varepsilon B)$, (1)

where " $\sqsubset$ " stands for "est" used in c)-case. It should be clear that the other way of using the copula should be reduced to this most basic copula " $\varepsilon$ ".

\section{$\S 4$ Ontological Law of Identity}

Now that we have at hand the axiom Ax.O. which governs the logical behavior of the purelly ontological copula " $\varepsilon$ ", we can pass on to the analysis of the "ontological law of identity". This law has the following expression;

O.L.I. $A$ est $A$, and it is said that it reflects the property of self-dependency of primary substances. Primary substances are those which depend on nothing other than self. And it is said that the ontological law of identity expresses this property of primary substances. But the logical sense and content of the law is by no means clear, when it is expressed in everyday language. It is entirely possible to take the law as expressing "whatever is $A$ is $A$ ", i.e. $(X)$ ( $X$ is $A \supset X$ is $A$ ), which is a mere logical tautology, and has no informative power, and is nonsensical in a sense. Such circumstances are easily caused, if the logical analysis of the copula "est" is not performed in a proper way. But, since we are now in possession of the notion of the purely ontological copula and Ax.0. which governs the logical behavior of the purely ontological copula, we are in a position to capture the logico-semantical content of the law whose sense and content is at first glance ambiguous and unclear. The logical intention of the ontological law of identity becomes clear, if we understand the "est" as " $\varepsilon$ "; we get indeed the following, if we substitute "est" in O.L.I. by " $\varepsilon$ ";

$$
\text { O.L.I* } \begin{aligned}
A \varepsilon A & \equiv(\exists X)(X \varepsilon A)_{\wedge}(X, Y)\left(X \varepsilon A_{\wedge} Y \varepsilon A \supset X \varepsilon Y\right) \\
& \equiv(\exists X)(X \varepsilon A)_{\wedge}(X, Y)\left(X \varepsilon A_{\wedge} Y \varepsilon A \supset X \varepsilon Y\right),
\end{aligned}
$$

which states that " $A$ est $A$ " is equivalent to "just one $A$ exists", i.e. " $A$ is an individual". To put it in other way, what the ontological law of identy purports to mean is the being (esse) simpliciter of the individuals. Hence,

T.O.I. $A \mathcal{E} A \equiv A$ is an individual, or, abbreviating " $\cdots$ is an individual" as "ob $(\cdots)$ ";

T.O.I.* $A \varepsilon A \equiv \mathrm{ob}(A)$.

(1) I borrow Lejewski's symbolism. Cf. Lejewski, C.; "On Lesniewski's Ontology", Ratio, Vol. 1, 1958. 150-176. Its Japanese translation by A. Ishimoto and S. Watanabe is in Ronrishiso no Kakumei (Revolution in Logical Thoughts), A. Ishimoto (ed.), 1972. To Japanese-reading readers, my article "Chokkan no Keishiki-ka to Ronriteki Sonzairon (Formalization of Intuition and Logical Ontology)", Philosophy, Mita Philosophical Society, No. 71, 1980, will be helpful. I wish to express my thankfulnoss to Prof. C. Lejewski for his having taken the trouble to give some valuable comments on some formulas in my above mentioned artiole, though it was written in Japanese. 
Without introducing the notion of purely ontological copula, we shall surely fail to capture the logical intention of the ontological law of identity, and it will be difficult for us to defend ourselves against the Kukasiewicz-type objection that the ontological law of identity is a mere play on words, or lauter Phrasenmacherei.(1)

\section{\$5 Categorial Reading of Quantifiers}

In the analysis of the ontological law of identity, we used the so-called referential reading of quantifiers ${ }^{(2)}$. As its opposite reading, we have the socalled substitutional interpretation of quantifiers. ${ }^{(3)}$ The reading of quantifiers will be as following; according to the referential interpretation,

R.I.Q. $\quad(x)(\cdots x \cdots)$; for every object $x ; \cdots x \cdots$

$(\exists x)(\cdots x \cdots)$; for some object $x ; \cdots x \cdots$,

while if we take the substitutional interpretation, the reading will be;

S.I.Q. $(x)(\cdots x \cdots)$; for every expression $x ; \cdots x \cdots$,

$(\exists x)(\cdots x \cdots)$; for some expression $x ; \cdots x \cdots$,

Each of them has its merits and demerits. If we take the referential interpretation, we can speak of objects, but we can quantify only the terms belonging to the syntactic category of "singular names", and the other syntactic parts we cannot quantify, for under this interpretation of quantifiers, to quantify the expressions belonging to a syntactic category means to require the existent abstract objects which are referred to by the expressions. A philosopher of nominalistic attitude cannot stand this position. The substitutional interpretation is immune from this danger, since the quantification is about "expressions", and quantifying expressions does not lead to the danger of Platonism. But what is troubelsome with this interpretation is that we cannot speak about objects by means of this interpretation.

I propose another interpretation of quantifiers which I call categorial interpretation of quantifiers. (4) This interpretation goes between the two interpretations mentioned above. It is an evident linguistic fact that the expressions belonging to whatever syntactic category have their specific logico-semantical function, which I call categorial convention of the expressions. The categorial convention of the

(1) Cf. p. 44

(2) Cf., e.g, Quine's "Designation and Existence", Journal of Philosophy, 36, 1939, 701709. "On what there is", reprinted in From a Logical Point of View, 1953. "Existence and Quantification", 1968, reprinted in Ontological Relativity \& Other Essays, 1969. Cf. also my "Ontological Burden of Grammatical Categories", The Annals of the Japan Association for Philosophy of Science, Vol. 5, No. 4, March.

(3) Cf. Marcus, R.B." "Interpreting Quantificution", Inquıry, vol. 5, 252-259.

(4) In my "Ontological Burden of Grammatical Categories", The Annals of the Japan Association for Philosophy of Science, Vol. 5, No. 4, March, I called it the "subjectivistic interpretation" of quantifiers. But in order to avoid unnecessary misunderstanding which can be caused by this terminology, I here call this way of interpretation the "categorial interpretation". 
expressions belonging to the "singular names" is that each of them refers to just one existing object. In short, what is characteristic to this interpretation is that a logico-semantic role is assigned to every syntactic category, and the logicosemantic role is embedded in the reading of quantifiers. It can be the case that the logico-semantical role of the expressions belonging to a semantic category is merely syncategorematic. In such a case, the categorial convention of the syntactic category is purely syntactical, and the substitutional interpretation will be used in reading quantifiers.

Instead of the referential reading, I wish to use hereafter the categorial reading of quantifiers. Making an essential use of the result of the previous section, i.e. T.O.I., I set up the following categorial convention of general names;

C.G.; $A$ general name indefinitely refers to objects which fall under its extension, where it can be either empty or just one or more than one. $A$ general name refers to just one individual if and only if with respect to it the ontological law of identity holds.

The general verbal reading of quantifiers read in the way of categorial interpretation will be as following. Suppose that the expressions of the category $\alpha$ have as their categorial convention $C(\alpha)$, then;

C.I.Q. $(x)(\cdots x \cdots)$; for every expression with the categorial convention $C(\alpha)$; $\cdots x \cdot \cdots$

$(\exists x)(\cdots x \cdots)$; for some expression with the categorial convention $C(\alpha) ; \cdots x \cdots$

Now for $C(\alpha)$, let us take C.G. Then the verbal reading of Ax.0. is; .

Ax.O.* $A$ is $B$ iff for some expression $X$ with the categorial convention C.G.:

$X$ is $A$, and for every expression $X$ and $Y$ with the categorial convention

C.G.: if $X$ is $A$ and $Y$ is $A$ then $X$ is $Y$, and for every expression $X$ with the categorial convention C.G.: if $X$ is $A$, then $X$ is $B$.

Now the followings are theorems deducible from Ax.O.;

T.1. $X \varepsilon A \supset X \varepsilon X$

Proof. 1. $X \varepsilon A$

(sup.)

2. $(\exists Y)(Y \varepsilon X)$

(1, Ax.0.)

3. $(Y, Z)\left(Y \varepsilon X_{\wedge} Z \varepsilon X\right.$. $\left.Y \varepsilon \varepsilon\right) \quad(1$, Ax.0.)

4. ( $Y)(Y \varepsilon X \supset Y \varepsilon X)$ (theorem)

5. $X \varepsilon X$

T.2. $(\exists X)(X \varepsilon A) \equiv(\exists X)\left(X \varepsilon X_{\wedge} X \varepsilon A\right)$

Proof. obvious from T.1.

T.3. $(X, Y)\left(X \varepsilon A_{\wedge} Y \varepsilon A . \supset X \varepsilon Y\right)$

$$
\equiv(X, Y)\left(X \varepsilon X_{\wedge} Y \varepsilon Y_{\wedge} X \varepsilon A_{\wedge} Y \varepsilon A . \supset X \varepsilon Y\right)
$$

Proof. obvious from T.1.

T.4. $(X)(X \varepsilon A X \varepsilon B) \equiv(X)\left(X \varepsilon X_{\wedge} X \varepsilon A . \supset X \varepsilon B\right)$ 
From these theorems and the categorial convention C.G., we obtain the following verbal reading of $\mathrm{Ax} . \mathbf{0}$.;

Ax.0.** $A$ is $B$ iff some individual is $A$, and at most one individual is $A$, and every individual $A$ is an individual $B$.(1)

Now we are in a position to quantify the names appearing in Ax.0. unbound, without falling into Platonism. I restate the Ax.O. in the following way;

Ax.0. $(A, B)\left(A \varepsilon B \equiv(\exists X)(X \varepsilon A)_{\wedge}(X, Y)\left(X \varepsilon A_{\wedge} Y \varepsilon A . \supset X \varepsilon Y\right)\right.$

of which the verbal reading is;

$$
\wedge(X)(X \varepsilon A \supset X \varepsilon B)),
$$

for every expression $A, B$ with the categorial convention $C . G .: A$ is $B$ iff some individual is $A$, and at most one individual is $A$, and every individual that is $A$ is an individual that is $B$. Hereafter I mean by Ax.O. the formula written just above, with its categorial interpretation of quantifiers, and use Ax.O. interpreted by the categorial interpretation as its official version.

\section{\$6 Cntological Law of Contradiction}

Having analysed the logico-semantical content of the law of identity, we are now in a position to analyse the logico-semantical structure and content of the ontological law of contradiction. I take the following two formulations as its verbal representation;

O.L.C.1 $A$ non est $B$ et non $B$

O.L.C.2 $A$ non est non $A$.

We need some logical preliminaries before analysing their logico-semantical structure, because as is clear at first glance, their logical structure is much more complicated by the fact that they contain negation "non" and the connective "et".

Logical Preliminaries.

I. On "names"; I understand by "name" "general name". When we describe the reality, we make use of names, or name-like expressions which we have in hand, or we create new names or name-like expressions, but in any case, when we use a name, at least one condition must be observed; namely the condition that they should be designative. The best example we make use of in forming a new namelike expression is "that which"; this forms a name-like expression by being attached to a verb. Anyway names or name-like expressions constructed by means of some lingustic device are used in support of the presupposition that they are designative.

(1) Ax.O. will be cexpressed, using T.O.I.* and T.2-T.4., as follows;

$A \varepsilon B \equiv .(\exists X)\left(o b(X)_{\Lambda} X \varepsilon A\right)_{\wedge}(X, Y)\left(o b(X)_{\Lambda} o b(Y)_{\wedge} X_{\varepsilon} A_{\wedge} Y \varepsilon A . \supset X_{\varepsilon} Y\right)$ ${ }_{\wedge}(X)\left(o b(X)_{\wedge} X_{\varepsilon} A \supset X_{\varepsilon} B\right)$, from which it is clear that only the variables over individuals are quantified. This vindicates the referential reading we took in 83 . 
We formulate this condition in the following way; let the name " $\alpha$ " be to be introduced into a discourse about what is really taking' place. Now the conditions of introducing the name " $\alpha$ " are 1 ) that there is at least one individual which satisfies the condition " $\lambda x \phi(x)$ ", (1) and 2) that the extension of " $\alpha$ " is identical with the extension of the individuals which satisfy the condition " $\lambda x \phi(x)$." So the general scheme of name-introduction of a name " $\alpha$ " with respect to a condition " $\lambda x \phi(x)$ " is of the following form;

O.D. $A \varepsilon \alpha \equiv o b(A)_{\wedge} \phi(A)$.

Rewriting "ob $(A)$ " by T.I.0*, we get;

O.D.* $A \varepsilon \alpha \equiv A \varepsilon A_{\wedge} \phi(A)$.

This is the general scheme for introducing names.

Now we introduce the expression "that which" by means of 0.D.*;

O.D.* $A \varepsilon \operatorname{trm}(F) \equiv A \varepsilon A_{\wedge} F(A)$, (2)

where the expression "trm $(\cdots)$ " corresponds to the everyday expression "that which...". Let " $F$ " be "run(s)". Then " $F(A)$ " is " $A$ run(s)", and "trm $(F)$ " is "that which run(s)".

II. On transforming a "predicative part" into a "verb"; our everyday language contains a device for transforming a predicative part of a sentence into a verb. As an example, let us take the following two expressions; 1) "Socrates est currens", and 2)" Socrates currit". The sentence 1) is convertible to the sentence 2). We may suppose that the following is taking place in this grammatical tranformation, namely; the predicative part of the sentence 1), i.e. "est currens" is transformed into the verb "currit". To put the matter formally, let us substitute the expression "Socrates" by " $A$ " and the expression "currens" by " $B$ ". Then sentence 1) will be;

\section{E.1. $A \varepsilon B$.}

What has taken place in the grammatical transformation is that the two components of the predicative part, i.e. " $\varepsilon$ " and "currens" were taken out as composing a grammatical unit, and the unit was transformed into the verb corresponding to " $B$ ". So, to put the matter formally, two logical steps are performed;

Step 1. composing a grammatical unit from the two grammatical basic elements, i.e. " $\varepsilon$ " and " $B$ ".

Step 2. converting the grammatical unit obtained by the step 1. into its corresponding verb.

(1) " $\lambda x \phi(x)$ " is a snetence-forming functor with one name-argument. Expressing the syntactic category of this functor according to Ajdukiewicz, it belongs the syntactic category of "s/n". Cf. Ajdukiewicz, K.: "Die syntaktische Konexität", Studia Philosophica, 1, 1-28.

(2) The parentheses " $(f)$ " indicates that the expressions which may be put between the parentheses belong to the category of verbs, i.e. " $s / n$ ". 
The operation consisting of these two steps, I shall express by the symbol " $f \neq$ ".(1) That is, the verb made out of a predicative part " $\varepsilon B$ " will be expressed as " $(\varepsilon B)$ ".

Since this transformation of "predicative part" into its corresponding "verb" is in general possible in our everyday language, let us codify this transformation in the following way;

$$
\text { P.D }{ }_{1}^{*} \quad(\varepsilon B)(A) \equiv A \varepsilon B \text {. }
$$

III. On "negation": In the standard text books of logic, only the sentential negation, which I express as " $\sim$ ", is treated. But our linguistic intuition tells us that at least two other sorts of negation exist; i.e. "nominal negation", and "predicative negation". Now that we have $0 . D^{*}$, we introduce the "nominal negation $(N)$ " in the following way;

$0 . \mathrm{D}_{2}^{*} \quad A \varepsilon N B \equiv A \varepsilon A_{\wedge} \sim(A \varepsilon B)$, the intuitive meaning of which is;

$A$ is non- $B$ iff $A$ is an individual and $A$ is not $B$, or

$A$ is non- $B$ iff $A$ is an individual which is not $B$. Thus we have introduced the notion of the "nominal negation."

In many of the sentences which are ususally expressed by means of sentential negation, the negation used in them is not a genuine sentential one, but "predictive negation". As an example, let us take a sentence "Scorates is not big". Putting "Socrates" as " $A$ " and "is big" as " $F$ ", the sentence will be expressed formally in the following way;

$$
\sim(F(A)) \text {. }
$$

But a short consideration tells us that what the sentence "Socrates is not big" purports to inform us is that a person designated by the name "Socrates" is not big, and the negation should be regarded as being involved as a part of the predicative part. Taking this fact into account, it should be clear that the proper expression of the expressions of the form " $\sim(F(A))$ " is to be expressed as " $(7 F)(A)$ ". (2)

Since it seems to hold generally for a sentence of the form " $F(a)$ " that a seemingly sentential negation in reality functions as a predicative negation, I codify this logical situation in the following way;

$$
\left.\mathrm{P.D}_{2}^{*}(\urcorner F\right)(A) \equiv \sim F(A) \text {. }^{(3)}
$$

(1) The parentheses " $f)$ " expresses the operation which operates on " $\varepsilon$ " and a name, and makes a one-place predicative. Its syntactic category is written in Ajdukiewicz-style as " $(s / n) /((s / n, n), n)$ ". Notice that a functor is introduced by the de nition P.D ${ }_{1}^{*}$.

(2) The expression " $\rceil$ " standsd for "predicative negation", the syntactic category of which is ' $(s / n) /(s / n)$ ', i.e. it is a functor which makes from a one-place predicative a one-place predicative.

(3) The proper introduction is the following;

P.D. $T<F \rightarrow(A) \equiv \sim F(A)$. But for the sake of easiness in understanding the logical intention, I use the informal de nition P.D. ${ }_{2}^{*}$. 
IV. On "compound" names: I wish here to reconfirm that we are in this paper concerned only with "general names". Now in our everyday language we have compound names like "a pretty and sweet-hearted girl" or "a pretty or sweethearted girl". There may be an argument that the expressions are not "compound names" but a name qualified by a "compound adjective". I suppose that this argument is correct. But in this paper, I take the position that they are compound names, interpreting them as "a pretty (girl) and sweet-hearted girl" and "a pretty (girl) and swett-hearted girl". To state the reason concisely, it is because an adjective should be regarded for an ontological reason as a "name".(1) In such a case as "Aristotle is a philosopher and scientist", there is no doubt that the connective "and" forms as a name-forming functor from two names. We codify the way of introducing compound names in the following way;

$$
\begin{aligned}
& \text { O. } \mathrm{D}_{3}{ }^{*} A \varepsilon(B \cap C) \equiv A \varepsilon B_{\wedge} A \varepsilon C \text {, } \\
& \text { O.D }{ }_{4}^{*} \quad A \varepsilon(B \cup C) \equiv A \varepsilon B^{\vee} A \varepsilon C^{(2)} \text {. }
\end{aligned}
$$

We thus introduce the compound names " $B$ et $C$ ", and " $B$ vel $C$ ".

After having finished these logicaly preliminaries I-IV, we are in a position to analyse the logical sense and content of the ontological law of contradiction.

Let $L o$ be the first-order language supplied with the following specific axioms and definitions;

Ax.0. $(A, B)\left(A \varepsilon B \equiv(\exists X)(X \varepsilon A)_{\wedge}(X, Y)\left(X \varepsilon A_{\wedge} Y \varepsilon A . \supset X \varepsilon Y\right)\right.$

Ax. $\phi . \quad(\phi)(\exists Z)(X)\left(X \varepsilon Z \equiv . X_{\wedge} \phi(X)\right)^{(3)}$

$$
\wedge(X)(X \varepsilon A \supset A \varepsilon B))
$$

Defintions; O.D ${ }_{1}^{*}-0 . D_{4}^{*}$

the introduction of which is legalized by "Ax. $\phi$ ", and in addition to them;

Definitions; P.D ${ }_{1}^{*}, \mathrm{P}^{\mathrm{D}} \mathrm{D}_{2}{ }^{*}$,

which are in their essence of propositional character.

Hereafter the analysis will be performed in $L o$.

I begin with the analysis of the ontological law of the first type. Its verbal form was;

O.L.C.1. $A$ non est $B$ et non $B$.

What is the intention of this ontological dictum? The point of the analysis of the

(1) Cf. Tbomas' commentary: Hoc autem nomen album significat subiectum, ex con. sequenti, inquantum significat albedinem per modum accidentis. Unde opportet, quod ex consequenti includat in sui ratione subiectum. (Italics by the present author) (In Meta., L.V., lec. IX. 894) On this point, of. Waragai, T.:' Formal characters of Aristotelian Language", Philosophy, Mita Philosophical Society, No. 69, 1979.

(2) Strictly following 0.D.*, "A $\varepsilon$ A" should appear in the definiens as its first component, but as was shown in T.l, this condition can be omitted.

(3) In order to restrict the order of logical types, I use "Ax.申." instead of O.D.*. Hereby, we are concerned with the first-order language. The categorical convention of " $\phi$ " is that it is of the syntactic category of ' $\mathrm{s} / \mathrm{n}$ '. 
law stated in the form O.L.C.1. is the analysis of logical function of the two negations in 0.L.C.1. and that of the connective "et". Let us restate O.L.C.1. in the following way in order to make the argument that follows perspicuous;

O.L.C.1.' $A$ non $_{1}$ est $B$ et non ${ }_{1} B$.

It seems to go without saying that "non," is the nominal negation which was introduced into Lo by P.D ${ }_{2}^{*}$ and the connective "et" is the logical connective introduced into $L o$ by means of the definition $0 . D_{3}{ }^{*}$. So the troublesome point in the analysis of O.L.C.1. is in the analysis of the logico-semantic function of "non 1 ".

Now if the ontological law of contradiction is intended to give some information about reality (more strictly speaking, about primary substances), then the subject term " $A$ " has to be (singular) designative, so that the analysis must be carried out in such a way that the subject term " $A$." is singular-designative, and at the same time in such a way that some ontological information as to what the term " $A$ " refers to can be given. As the circumstances are so, it is clear that the copula "est" in O.L.C.1. should be understood as the purely ontological copula, and since "non " cannot be nominal negation, we have two possible interpretations as to this negation-particle. We can take it either 1) as sentential negation, or 2) as predicative negation.

Now let us take it as sentential negation. Then O.L.C.1, can be expressed in $L o$ in the following way;

$$
\text { O.L.C.1* } \sim(A \varepsilon(B \cap N B)) \text {. }
$$

Now in $L o$, O.C.L. F $^{*}$ is a theorem. Indeed;

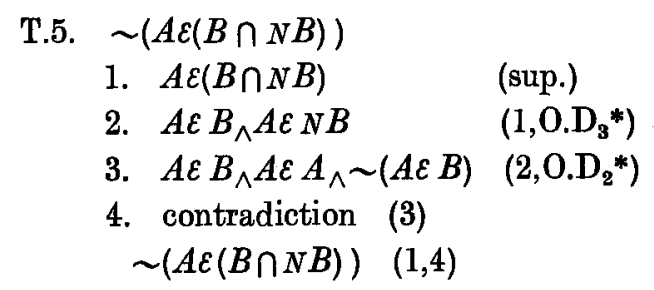

That O.L.C.1* becomes a theorem in $L o$ when we take the negation-particle 'non,' as sentential means that the ontological law of contradiction is in this interpretation a tautology, and it states nothing about what the term " $A$ " refers to, which in turn is tantamount to being ontologically nonsensical in the sense that it gives us no ontological information. Hence the negation-particle "non," cannot be sentential.

Let us now investigate the second possible case according to which the negationparticle in question is predicative. In this case, it is clear that the predicative part which is to be negated is "est $B$ et non $B$ ". Translating this into $L o$, we obtain the following;

E.2. $(\varepsilon(B \cap N B))$

From this, we obtain the following, making use of the predicative negation; 
E.3. $\urcorner(\varepsilon(B \cap N B))$.

In order to connect this with the term " $A$ " by means of " $\varepsilon$ ", we need to transform this negative predicative part into a name, so that we transform " $T\{\varepsilon(B \cap N B)$ )" into "trm $(T € \varepsilon(B \cap N B)))$. As a result of this analysis, we obtained the following formula when we take "non 1 " as predicative negation;

O.L.C.1** $A \varepsilon \operatorname{trm}(T(\varepsilon(B \cap N B)))$, that is, the normal grammatical form of the ontological law of contradiction of the form O.L.C.1 is;

$A$ est id quod non est $B$ et non $B$,

and O.C.L.1. is its grammatically abbreviated form. As for this the following is a theorem;

T.6. $A \varepsilon \operatorname{trm}(\rceil(\varepsilon(B \cap N B))) \equiv A \varepsilon A$.

1. $A \varepsilon \operatorname{trm}(\rceil\{\varepsilon(B \cap N B))) \equiv A \varepsilon A_{\wedge}(\rceil\{\varepsilon(B \cap N B) \xi)(A) \quad\left(0 . \mathrm{D}_{1}^{*}\right)$

2. $\quad \equiv A \varepsilon A_{\wedge} \sim(A \varepsilon(B \cap N B)) \quad\left(1, P^{2} . D_{2}^{*}\right)$

3. $\quad \equiv A \varepsilon A_{\wedge} \sim\left(A \varepsilon B_{\wedge} A \varepsilon N B\right) \quad\left(2,0 . D_{3}^{*}\right)$

4. $\quad \equiv A \varepsilon A_{\wedge} \sim\left(A \varepsilon B_{\wedge} A \varepsilon A_{\wedge} \sim(A \varepsilon B)\right) \quad\left(3,0^{2} D_{2}^{*}\right)$

5. $\equiv A \varepsilon A$

This states that the ontological law of contradiction gives us an ontological information as to what the term " $A$ " refers to, i.e.; " $A$ is an individual being."

We now pass on to the analysis of the ontological law of contradiction which is expressed in the form O.L.C.2., i.e.;

O.C.2. $A$ non est non $A$.

I restate this as follows in order to make it easier to speak about its logicosemantic structure;

O.L.C.2'. $A$ non $_{1}$ est non $_{2} A$.

In this case, too, there are two clear points, i.e. 1) the negation-particle "non ${ }_{2}$ " is nominal, and 2) the copula "est" is the purely ontological cupla, so that the whole problem depends on whether we take the negation-particular "non" as sentential or predicative.

Now let us take it as sentential. Then O.L.C.2. takes in Lo the following form;

L.O.C.2* $\sim(A \varepsilon N A)$,

which is, however, a tautology in $L o$.

T.7. $\sim(A \varepsilon N A)$

1. $A \varepsilon N A$

2. $A \varepsilon A_{\wedge} \sim(A \varepsilon A) \quad\left(1,0 . D_{2}^{*}\right)$

3. contradiction

$\sim(A \varepsilon N A)$

so that to interpret the negation-particle as sentential leads us to the same situation we faced to when we analysed O.L.C.1. As was the case in the analysis of 
O.L.C.1., the ontological law of contradiction in the form O.L.C.2. in this interpretation is reduced to a mere tautology which tells nothing about what the term " $A$ " refers to, and becomes nonsensical in the sense that it gives ontologically no information. Hence, this negation cannot be sentential.

Now let us take this negation-particle as predicative negation. Then, the predicative part to be negated is evidently "est non A", so by translating this into $L o$, we obtain the following;

E.4. $(\varepsilon N A)$, from which we obtain, using predicative negation, the following;

E.5. $\rceil\{\varepsilon N A)$.

Further, in order to connect this with the term " $A$ " by means of " $\varepsilon$ ", we convert E.5. to a name;

E.6. $\operatorname{trm}(\rceil(\varepsilon N A))$.

As the result, we obtain the following;

O.L.C.2** $A \varepsilon \operatorname{trm}(T(\varepsilon N A))$.

From this, the normal form of the ontological law of contradication expressed in the form O.L.C.2. is verbally as following;

$A$ est id quod non est non $A$,

and the verbal expression of the ontological law of contradiction O.L.C.2. is a grammatical abbreviation of the sentence mentioned above. As for this, the following holds;

T.8. $A \varepsilon \operatorname{trm}(T(\varepsilon N A)) \equiv A \varepsilon A$

1. $\left.A \varepsilon \operatorname{trm}(\rceil\{\varepsilon N A) \equiv A \varepsilon A_{\wedge}\right\rceil \varepsilon N A(A) \quad\left(\right.$ O.D.1 $\left.{ }^{*}\right)$

2. $\quad \equiv A \varepsilon A_{\wedge} \sim(\varepsilon N A(A)) \quad\left(1,{\mathrm{P} . \mathrm{D}_{2}}^{*}\right)$

3. $\quad \equiv A \varepsilon A_{\wedge} \sim(A \varepsilon N A) \quad\left(2, \mathrm{P}^{\circ} \mathrm{D}_{1}^{*}\right)$

4. $\quad \equiv A \varepsilon A_{\wedge} \sim\left(A \varepsilon A_{\wedge} \sim(A \varepsilon A)\right) \quad\left(3,0 . \mathrm{P}_{2}{ }^{*}\right)$

5. $\equiv A \varepsilon A \quad$ (4).

This means that the interpretation of the negation-particle as predicative gives us an ontological information about what the term " $A$ " refers to, namely; " $A$ is an individual being."

From these analyses, we obtain the following general conclusion:

1. There is an adequate system in which the logico-semantical analysis of the ontological law of identity and the ontological law of contradiction can be performed, i.e. the language we called $L o$.

2. In these ontological theses, there appears no sentential negation.

There appear in them solely nominal negation and predicative negation.

3. Both the ontological law of identity and the ontological law of contradiction ontologically express the same state of affairs, i.e. esse simpliciter of individual beings. 


\section{§7 System Lo and Leśniewski's Ontology}

Though the logico-semantical analysis of Aristotle's argument of descending chain of predicates which is essentially ontological, and at the same time through the analysis of the everyday usage of names and negations, I made clear the logico-semantical content of the ontological law of identity and that of the ontological law of contradiction by constructing a first-order language $L_{0}$ which is strong enough to perform the logico-semantical analysis of the two ontological dicta. Historically speaking, this system is a proper part of a more comprehensive logical system constructed by Stanisław Leśniewski (1886-1939), which he named Ontology. (1)

He stated;

I used the name Ontology for the system I constructed, since, when I consider the circumstances that I formulated in the system a kind of "general principle of being", the name did not hurt my "feeling of language"(2).

But what we regret is that he does not seem to have mentioned any philosophical relation between his system Ontology and traditional Ontology.

In this paper, we obtained logical system through a philosophical and logical analysis of Aristotle's argument of descending chain of predicates, and it became clear that the analysis leads us to a logical system which is of its essential nature Leśniewskian. This fact helps understand the philosophical relation between Leśniewski's Ontology and traditional Ontology.

(1) The axiom of Ontology was found during the summer semester in 1919/1920, and officially announced in 1921. On this point; of. Lesniewski, S.: 'O Podstawach Matematyki', Rozdzial XI ('On the Foundations of Mathematics', Chapter XI). Przeglad Filozoficzny, 34, 1931. Its Japanese translation is in The Philosophy of Science, 1980, 89-102, translated by the present author. About Lesniewski's Ontology, cf. Lejewski, C.: "On Lesniewski's Ontology", Ratio, Vol. 1, 150-176. Slupecki, J.: "S. Leśniewski's Calculus of Names", Studia Logica, Vol. 3. Also cf. Henry, D.P.: Medieval Logic and Metaphysics, 1972.

(2) p. 163 of Leśniewski's work mentioned in (1). 